\title{
Percepción táctil, visual y auditiva en niños víctimas de maltrato intrafamiliar
}

\author{
Táctil, visual and auditory perception \\ in children victims of domestic abuse
}

Martha Cobos Cali ${ }^{1}$

mcobos@uazuay.edu.ec

\author{
Valentina Ladera Fernández ${ }^{2}$ \\ ladera@usal.es \\ María Victoria Perea Bartolomé ${ }^{3}$ \\ vperea@usal.es \\ Ricardo García García ${ }^{4}$ \\ rigar@usal.es
}

\begin{abstract}
Resumen
Este trabajo analiza la percepción táctil, visual y auditiva en niños víctimas de maltrato intrafamiliar. Participaron 104 niños divididos en dos grupos. El primero conformado por niños institucionalizados por violencia intrafamiliar (NVVI); el segundo por niños que no han sido víctimas de violencia (NNVI), equiparados en género, edad y escolarización. Se usaron subescalas de percepción visual, auditiva y táctil de la evaluación neuropsicológica infantil. Los resultados muestran que los NVVI presentan menor rendimiento en la tareas de reconocimiento de expresiones; sonidos ambientales; $y$ en percepción fonémica. En estos niños se encuentra conservada la percepción táctil; la percepción visual es adecuada excepto cuando se involucra el componente emocional; $y$ en lo auditivo existe déficit en percepción de sonidos ambientales y verbales.
\end{abstract}

\section{Palabras clave}

Maltrato infantil, violencia, percepción visual, auditiva y táctil.

Forma sugerida de citar: Cobos Cali, Martha, Ladera Fernández Valentina, Perea Bartolomé, María Victoria \& García García, Ricardo (2016). Percepción táctil, visual y auditiva en niños víctimas de maltrato intrafamiliar. Universitas, XIV(25), pp. 169-190.

1 Profesora titular de la Facultad de Filosofía, Letras y Ciencias de la Educación de la Universidad del Azuay.

2 Profesora titular de la Facultad de Psicología de la Universidad de Salamanca.

3 Profesora titular de la Facultad de Psicología de la Universidad de Salamanca.

4 Profesor titular de la Facultad de Psicología de la Universidad de Salamanca. 


\begin{abstract}
The aim of this paper is to analyze the táctil, visual and auditory perception in children victims of domestic abuse. 104 children participated divided into two groups. The first group made up of children institutionalized by domestic violence (NVVI); the second made up of children who have not been victims of violence (NNVI), matched for gender, age and schooling. Subscales of visual, auditory and tactile perception child neuropsychological assessment were used. The results show that the NVVI have lower performance in tasks: recognition of expressions; ambient sounds; and phonemic awareness. The study is inferred that child victims of domestic violence recognition of expressions is altered, also in this group of children it is deficient perception of environmental and speech sounds. In these children is preserved tactile perception; visual perception is adequate except when the emotional component is involved; and auditory perception deficit exists environmental and verbal sounds.
\end{abstract}

Keywords

Child abuse, domestic violence, táctil, visual and auditory perception.

\title{
Dificultades en la percepción auditiva y visual en niños víctimas de maltrato intrafamiliar
}

Desde sus orígenes la humanidad ha tenido dificultades con dolorosas manifestaciones de violencia en lo personal, familiar y en los diversos ámbitos sociales. La Organización Panamericana de la Salud (2002) en su informe sobre violencia señala que la Organización Mundial de la Salud (OMS) en 1996 declaró la violencia como un importante problema de salud pública a nivel mundial.

Se define a la violencia de la siguiente forma:

El uso deliberado de la fuerza física o el poder, ya sea en un grado de amenaza o efectivo, contra uno mismo, otra persona o un grupo o comunidad, que cause o tenga muchas probabilidades de causar lesiones, muerte, daños psicológicos, trastornos del desarrollo o privaciones. La definición comprende tanto la violencia interpersonal como el comportamiento suicida y los conflictos armados (OPS, 2002, p. 4). 
Se incluye en este concepto a la violencia intrafamiliar o de pareja, que en la mayor parte de los casos se produce entre miembros de la familia o compañeros sentimentales, y suele acontecer en el hogar, aunque no exclusivamente.

En la Constitución del Ecuador, en el Artículo No. 2 de la Ley contra la Violencia a la mujer y la familia, se define concretamente a la Violencia Intrafamiliar:

... toda acción y omisión que consista en maltrato físico, psicológico o sexual, ejecutado por un miembro de la familia en contra de la mujer o demás integrantes del núcleo familiar (Congreso Nacional. El plenario de las Comisiones Legislativas, 2008).

Dentro de este concepto varios autores han recalcado que la violencia intrafamiliar afecta directamente a los grupos más vulnerables, entre ellos, los niños y niñas (Calle, 2012; Corsi, 2006; Ojeda, 2010; OPS, 2014). La violencia hacia los niños se manifiesta a través del maltrato infantil, definido en los siguientes términos:

Los abusos y la desatención de que son objeto los menores de 18 años, e incluye todos los tipos de maltrato físico o psicológico, abuso sexual, desatención, negligencia y explotación comercial o de otro tipo que causen o puedan causar un daño a la salud, desarrollo o dignidad del niño, o poner en peligro su supervivencia, en el contexto de una relación de responsabilidad, confianza o poder. La exposición a la violencia de pareja también se incluye a veces entre las formas de maltrato infantil (OMS, 2014,parr.1).

De igual manera, el maltrato infantil ha sido conceptualizado en la Constitución del Ecuador en su Artículo No. 67 del Código de la Niñez y Adolescencia:

...toda conducta, de acción u omisión, que provoque o pueda provocar daño a la integridad o salud física, psicológica o sexual de un niño, niña o adolescente, por parte de cualquier persona, incluidos sus progenitores, otros parientes, educadores y personas a cargo de su cuidado; cualesquiera sea el medio utilizado para el efecto, sus consecuencias y el tiempo necesario para la recuperación de la víctima. Se incluyen en esta calificación el trato negligente, o descuido grave o reiterado en el cumplimiento de las obligaciones para con los niños, niñas y adolescentes, relativas a la prestación de alimentos, atención médica, educación o cuidados diarios; y su utilización en la mendicidad (Congreso Nacional del Ecuador, 2008). 
Se recalca, en base al texto antes descrito, que el concepto de maltrato infantil incluye el daño ocasionado dentro del hogar y en muchos casos por sus propios progenitores, es decir, originado en la violencia intrafamiliar.

Lamentable situación que en pleno siglo XXI aún no ha sido superada, $\mathrm{y}$ en el año 2015 Tsavoussis, Stawicki y Papadimos alertan acerca de una posible epidemia de niños víctimas de violencia doméstica; específicamente, aclaran que entre el 30 y $40 \%$ de personas implicadas en la violencia de género tienen hijos y, por lo tanto, hay 275 millones de infantes testigos de fuertes agresiones dentro de sus hogares. Por su parte, UNICEF (2014) aseveró que en el mundo existen entre 500 y 1500 millones de niños víctimas violencia.

En el Ecuador, según su Secretaría Nacional de Planificación y Desarrollo (SENPLADES, 2012), la situación no es diferente: un total de 4553 niños maltratados fueron atendidos en centros de acogida en todo el país y concretamente en la ciudad de Cuenca, en el año 2012, se atendieron aproximadamente 355 niños mensualmente. Estos niños, víctimas de maltrato intrafamiliar, que generalmente incluye la exposición a una violencia constante desde etapas muy tempranas, e inclusive se estructura en una forma trans-generacional, su entorno provocará inevitable y forzosamente un efecto marcadamente negativo.

Al igual que a nivel mundial, en el Ecuador existen hitos importantes en la lucha en contra de la violencia de la familia, Ojeda (2010) recoge los siguientes: la creación de la Oficina Nacional de la Mujer (1979), la inauguración de la Casa Refugio para Mujeres y Menores Maltratadas y la reforma del Código Civil (1989), la publicación de la Ley 103 contra la violencia a la mujer y la familia (1989), el reconocimiento de la violencia contra las mujeres en el ámbito familiar como violación a los derechos humanos y el reconocimiento de la violencia contra la mujer como un problema de salud pública (1998); también hay que mencionar la creación de las comisarías de la mujer (2000), la publicación del reglamento a la Ley contra la Violencia a la Mujer y Familia, el Decreto de la Erradicación de la Violencia de Género como política de Estado y la elaboración del Plan Nacional de Erradicación de la Violencia de Género (2004).

Gavilanes (2008) registra que la preocupación por la niñez en el Ecuador empieza a darse en la década de los 80. En los años 90 se inició un movimiento social en favor de la infancia, en el cual se considera al niño como ciudadano y sujeto de derechos. En estos años se crea el Programa Operación Rescate Infantil (ORI) y el Fondo de Desarrollo Infantil (FODI). En el 
2004 se publica la "Aprobación del Plan Nacional Decenal de Protección Integral a favor de niños, niñas y adolescentes"; en el 2005 la "Protección infantil" fue establecida como política de Estado; a finales del 2005, 45 de los 219 Municipios conformaron Concejos Cantonales de la Niñez y Adolescencia, que estaban integrados por representantes gubernamentales y de la sociedad civil, responsables de políticas públicas a nivel local; en el 2008, el cambio en la Constitución del Ecuador busca proteger los derechos de los niños y adolescentes en situación de riesgo y proteger la institución familiar. A partir de esta Constitución se elabora el plan de protección integral a la niñez y adolescencia del cantón Cuenca 2008-2020 (Consejo Cantonal de la Niñez y Adolescencia de Cuenca y Alcaldía de Cuenca, 2008) y en cuya política de protección especial afirma:

Frente a situaciones de amenaza o violación de derechos como: maltrato, abuso, desaparición, explotación sexual, explotación laboral y económica, tráfico y trata, privación del medio familiar, adolescentes en conflicto con la ley, desplazados, refugiados, discapacidades, adolescentes embarazadas, hijo e hijas de migrantes, hijos e hijas de padres y madres privados de su libertad, entre otros; niños, niñas y adolescentes acceden a servicios de protección especial que garantizan la exigibilidad y restitución de sus derechos amenazados o violentados (p. 69).

Para cumplir con estos objetivos se dispone la política 13, en donde se busca:

Asegurar la temporalidad de la medida de acogimiento institucional y priorizar el desarrollo de alternativas de trabajo con familias y comunidad, para fortalecer sus capacidades de contención social y de restitución de derechos de niños, niñas y adolescentes (p.72).

El acogimiento institucional se ejecuta en caso de haber identificado a una persona en situación de amenaza o violación de sus derechos; generalmente la denuncia puede ser receptada por la Junta Cantonal de Protección de Derechos-Gobierno Autónomo Descentralizado de Cuenca y/o la Dirección Nacional de Policía Especializada en la Niñez y Adolescencia (DINAPEN) que se encargan de investigar. Si se considera necesario, el caso es enviado a los juzgados de la niñez y adolescencia para que sea un juez quien dictamine el procedimiento a seguir. Pueden intervenir otros actores como la Fiscalía General del Estado, la Defensoría del Pueblo, la Defensoría Pública y el Consejo Nacional de la Judicatura. 
Al identificar un niño vulnerado en sus derechos, por dictamen del juez es enviado a una casa de acogimiento institucional temporal, denominado Centro Municipal Integral de Servicios Sociales Solidarios (CEMISOL), y se interviene con la familia para buscar su reinserción; en el caso de no lograrse este objetivo en tres meses se lo remite a una segunda casa de acogida, que puede ser de carácter temporal o permanente, y en donde podrá permanecer el tiempo requerido. El dictamen de este acogimiento es facultativo, únicamente, del juez.

En el plan de protección integral a la niñez y adolescencia 2008-2013, se planteó como meta la implementación de un programa cantonal de acogimiento familiar para evitar que los niños sean asignados a una institución, o por lo menos lograr que el tiempo máximo de permanencia sea de tres años; sin embargo, esto no se ha logrado en su totalidad. El acogimiento institucional dependerá del tipo de casa de acogida y de la disponibilidad de cupos en estos lugares y le corresponde a CEMISOL su ubicación.

Las siguientes instituciones son las que obtuvieron autorización de funcionamiento otorgado por el Consejo Cantonal de la Niñez y Adolescencia de Cuenca y Alcaldía de Cuenca, (CCNA, 2013):

- Fundación Hogar del Ecuador "La Esperanza Hogares Para Niños": acogimiento a niños entre 6 y 12 años.

- Hogar de Niños Antonio Valdivieso: acogimiento a varones entre 6 y 12 años.

- Centro El Buen Pastor: acogimiento institucional para los adolescentes. Fundación el Jardín de El Cajas: acogimiento institucional a niños y adolescentes en situación de callejización.

- Fundación María Amor: acogimiento a mujeres víctimas de violencia y a sus hijos e hijas.

- Fundación Mensajeros de la Paz: acogimiento institucional de varios tipos.

- Hogar Miguel León: acogimiento institucional a niñas.

- Centro Municipal Integral de Servicios Sociales Solidarios (Cemisol): acogimiento temporal a niños, niñas y adolescentes en situaciones de amenaza o vulneración de derechos.

- Aldeas infantiles SOS: institución a nivel mundial de acogimiento a niños, niñas y adolescentes. 
- Fundación OSSO: acogimiento institucional a niños con capacidades especiales.

- Conferencia San Vicente de Paúl (Orfanato Tadeo Torres): acogimiento institucional a niños de 0 a 6 años.

La institucionalización no logra resolver el problema y desde la perspectiva de los niños que viven violencia intrafamiliar, este tipo de acogimiento constituye nuevamente un riesgo de sufrir un nuevo tipo de violencia, la violencia institucional (Litrownik, Newton, Mitchell y Richardson, 2003).

La institucionalización ha logrado cubrir la necesidad de vivienda, salud, educación, que si bien son fundamentales para la supervivencia de estos niños, no garantiza que ellos superen las secuelas emocionales, cognitivas y sociales que deja la experiencia de abandono y el sentimiento de desamparo (Obando, Villalobos y Arango, 2010, p. 152).

Según Morelato (2011), en la realidad se observa que la medida protectora de separar al niño de su familia maltratadora, probablemente sea un factor de protección, pero no de resiliencia, por lo que hay que mejorar los procedimientos utilizados a nivel institucional para lograr la felicidad del niño víctima de violencia; si no se logra una intervención adecuada, las consecuencias para los niños pueden ser irreversibles.

Manga y Fournier (1997), Manga y Ramos (2011), en base a las aportaciones de Reitan (1984), Luria (1978), identifican varias características de la neuropsicología infantil. Ante todo sobresale que la disfunción cerebral en niños es capaz de generar dificultades para adquirir nuevas habilidades cognitivas y capacidades comportamentales, mientras que en el adulto se expresará en fallos de capacidades ya aprendidas: "la lesión de una determinada zona de la corteza cerebral en la infancia afecta al desarrollo de zonas superpuestas a ella, mientras que la lesión de esa misma región en la edad adulta afecta, por el contrario, a zonas más internas del cerebro" (Vygotsky, 19281997d, p. 448), ley que ha sido elemento referencial en varios estudios posteriores (Giza, Mink y Madikians, 2007; Luria, 1978; Quintanar et al. 2008).

En relación al daño cerebral, síndromes idénticos pueden ser el resultado de una localización diferente de las lesiones cerebrales en el niño y en el adulto y, a su vez, lesiones con idéntica localización en niños y adultos pueden originar trastornos muy diferentes. También los trastornos cerebrales de los niños suelen ser más generalizados que focales, por lo que no suelen permitir una localización anatómica precisa. 
Finalmente, es difícil identificar el estado premórbido en el caso de los niños y el grado de déficit, además, del modo en que el daño cerebral afectará y desviará al niño en su futuro desarrollo y capacidad de aprendizaje.

Con estas características resaltadas se comprende que el estudio de la neuropsicología infantil implique el análisis de las funciones cognitivas en desarrollo. Las funciones psíquicas buscan la adaptación del sujeto a su medio y se las debe comprender en realidad como sistemas funcionales: "El substrato cerebral de los procesos psíquicos no lo integran sectores aislados, sino complejos sistemas de todo el aparato cerebral" (Vygotsky, 19821997i, p. 90).

Sin embargo, el sustrato anatómico cerebral es la base material para la posible existencia de los sistemas funcionales, pero no una garantía para su formación. Los sistemas funcionales complejos en los niños no aparecen ni se forman por sí solos, sino que se conforman a partir de las tareas o acciones que el niño realiza; así, únicamente con la formación de una acción, que es la unidad de análisis psicológico, se garantiza la conformación de su sistema funcional complejo correspondiente (Luria, 1978; Quintanar et al., 2008).

Johnson (2011) propone que existen tres teorías para comprender el desarrollo de las funciones cognitivas: la teoría de la maduración, la del aprendizaje y la de la especialización interactiva. Este autor pone de manifiesto las limitaciones de la teoría de la maduración y el aprendizaje, enfatizando que aisladamente no explican los procesos de desarrollo y, en consecuencia, considera necesario dar una explicación más completa.

Por lo que respecta a la teoría de la especialización interactiva, Johnson (2011) entiende que durante el desarrollo post-natal cambian las propiedades de respuesta de algunas regiones corticales. Las nuevas competencias conductuales durante la infancia serán asociadas con los cambios en la actividad de algunas regiones (redes) cerebrales y no solo con el inicio de esta actividad en una u otra región adicional. La especialización interactiva, además, se caracteriza por las redes neuronales, procesos de segregación e interacción, de plasticidad cerebral y de la causalidad circular, que incluye la epigenética como punto de vista del desarrollo.

En este proceso la relación que el niño establece con los primeros cuidadores es fundamental. Estos vínculos tienen la capacidad de modelar, ajustar y reorganizar las tendencias genéticas, es decir, ciertos componentes genéticos podrían ser activados o no, dependiendo de la interacción del niño con los adultos, proceso que se conoce como epigenética: "De ese modo, lo que se ex- 
perimenta en los primeros años de vida determina el futuro de cada persona y, por lo tanto, el futuro de la sociedad" (National Scientific Council on the Developing Child, 2010). Si bien cuando los estímulos se presentan demasiado tarde en la vida, pueden producirse pérdidas o carencias en el desarrollo; en caso contrario, si se anticipan pueden generar un estrés que interferirá con el desarrollo emocional (Gunnar, et al., 2009; Shonkoff, et al., 2013).

Los procesos cognitivos se basan en el funcionamiento de la actividad de sistemas cerebrales. En la ontogenia el desarrollo de este sistema depende de factores endógenos (genética) y de factores externos (medio ambiente). Las modificaciones, como el crecimiento de las neuronas y del funcionamiento neuronal, se relacionan con los cambios cualitativos, que incluyen la diferenciación celular, la transformación de las conexiones neuronales y los cambios de la organización del sistema funcional. Los periodos de reordenamiento cualitativo de los sistemas cerebrales son más sensibles a los factores medioambientales (Bezrukikh, Machinskaya y Farber, 2009).

La arquitectura básica del cerebro humano se desarrolla antes de que el niño nazca, cuando las neuronas se han organizado, formando la corteza y otras estructuras importantes del cerebro (Oates, Karmiloff-Smith y Johnson, 2012). Al nacer el niño, la corteza todavía muestra un grado de plasticidad considerable y las dimensiones de algunas áreas funcionales pueden aumentar o disminuir, según las pautas que rijan las vivencias prácticas y las experiencias del niño (Sur y Rubenstein, 2005). El desarrollo de los distintos sistemas cerebrales es variable y maduran con ritmos diferentes (Lebel y Beaulieu, 2011). Después del nacimiento del niño, se origina inicialmente una producción exuberante de conexiones cerebrales, seguida de una poda sistemática de conexiones, hasta formar un conjunto de redes cerebrales estables. Las experiencias del niño desempeñan un rol esencial a la hora de determinar qué sistemas se estabilizarán y cuáles dejarán de ser importantes desde el punto de vista funcional.

Las áreas primarias sensoriales y motrices de la corteza cerebral inician su proceso de mielinización antes que las áreas de asociación frontal y parietal, que alcanzan su desarrollo completo hacia los 15 años. Las fibras comisurales de proyección y de asociación completan su mielinización en una época más tarde que las áreas primarias (Roselli, Matute, Ardila, 2010). Las regiones prefrontal y parietal del cerebro interactúan con otras redes para facilitar su especialización y afinación cada vez mayores respecto a los estímulos ambientales (Johnson, 2011). 
En la formación del sistema visual, algunos de los estímulos que este necesita se producen cuando los bebés están todavía dentro del vientre materno. Después del nacimiento comienza la estimulación visual proveniente del mundo exterior. La estimulación visual también es fundamental para otros aspectos de la vista, como la sensibilidad a los contrastes, la percepción del movimiento y el procesamiento de los rostros. De todos modos, la ubicación temporal y la duración del período en que es necesario un flujo normal de informaciones visuales varían mucho, oscilando desde los pocos meses después de nacer hasta más allá de los diez primeros años de vida. Inclusive, cuando se alcanza un nivel maduro de funcionamiento, existe un período ulterior de estabilización durante el cual hacen falta normales experiencias visuales para poder mantener el nivel de competencia adquirido (Fox y Shonkoff, 2011). Estos autores piensan que este largo período de desarrollo permite que el sistema visual del niño se adapte a las características específicas de su ambiente.

Las informaciones visuales son importantes, además, para el desarrollo de otras áreas. La vista desempeña un papel significativo en las primeras habilidades sociales que, a su vez, influencien el desarrollo de otras habilidades como el lenguaje (Oates, Karmiloff-Smith y Johnson, 2012).

En una etapa sucesiva del desarrollo, las buenas capacidades de atención brindan también a los niños de preescolar una ventaja en las operaciones aritméticas y en la alfabetización, permitiendo predecir cuáles serán sus resultados al llegar a la escuela. Y posteriormente, cuando les toque modular la memoria de trabajo (Astle y Scerif, 2011).

En relación a la audición, los neonatos son capaces de distinguir todos los sonidos de todos los idiomas del mundo, aunque no los hayan oído nunca antes. Sin embargo, al terminar el primer año de vida, solamente conservan la habilidad de distinguir los sonidos que han escuchado en las conversaciones de las personas que los rodean (Werker y Tees, 2005).

La diversidad de oportunidades para la comunicación verbal con un cuidador habitual influye sobre el desarrollo del lenguaje del niño y sobre la adquisición de habilidades de lectoescritura, y estas habilidades predicen el logro académico en una etapa posterior de la vida. Puesto que las nuevas destrezas se desarrollan a partir de las anteriores, las primeras son las bases para el desarrollo infantil (Heckman, 2007). Además, las destrezas que dependen de la experiencia, como la capacidad para leer, descansan sobre la disponibilidad de experiencias claves de aprendizaje y de apoyo por parte del adulto (Shonkoff y Richter, 2013). 
A lo largo del desarrollo se ha observado que existen periodos críticos especialmente sensibles para el desarrollo cerebral y para la adquisición de funciones cognitivas de nivel más elevado (Knudsen et al., 2006). Epstein en 1986 afirmó que el crecimiento madurativo cortical ocurre por ráfagas u oleadas de 3 a 4, 6 a 8, 10 a 12 y de 14 a 16 años lo cual concuerda con la observación de que "el desarrollo cognitivo del niño tampoco sigue una línea uniforme, sino que es común que se presenten momentos de revelación (insight)" (Roselli, Matute, Ardila, 2010, p.19).

Es interesante para una mejor comprensión del desarrollo infantil conocer los tres niveles básicos de conducta en el proceso de la ontogénesis cerebral para la construcción de la cognición cerebral (Muñoz y Palau, 2004).

Nivel reactivo: Es la paleoconducta, la propiedad esencial del ser vivo, la excitabilidad, la conducta más primitiva del comportamiento, que se sustenta en estructuras troncomedulares; a este tipo de conducta pertenecen los reflejos, las sinergias y los automatismos.

Nivel propositivo: Se localiza en estructuras rinencéfalo-hipotálamo- talamocorticales, que dan lugar a un comportamiento mucho más complejo que utiliza patrones sensoriomotores capaces de mejorar con el aprendizaje. Su estructura funcional será el somatograma (esquema corporal), base del aprendizaje, que informa al organismo de sus relaciones físicas temporoespaciales con el mundo que lo rodea.

El recién nacido sufre una confusión total entre el mundo interior y exterior; la primera coordinación entre ambos se esboza madurativamente antes del cuarto mes, y alcanza su maduración completa al noveno. Entre los seis y nueve meses es capaz, en condiciones normales, de separar el espacio que lo rodea de su propio cuerpo. A partir de los 12 meses, la unidad corporal se construye a expensas de la representación visual.

El lenguaje se construirá mediante su programa genético al interaccionar con los primeros sonidos recibidos del medio ambiente y modularse mediante otros procesos. Su proyección constructiva se realizará cronológicamente, siguiendo de forma rigurosa los niveles de conducta madurados: reactivo, propositivo y comunicativo, mediante la mímica, el gesto y las fonías.

Entre los 4 y 5 años de edad se construye la lateralización.

En la formación del somatograma intervienen las vías de la sensibilidad propioceptiva, vestibular y dolorosa, el tálamo y las estructuras funcionales talamocorticales y corticotalámicas. 
Nivel comunicativo: Finalmente, en la construcción normal del niño, se establecería un nivel de conducta comunicativo a través de la empatía y la actividad expresiva, principalmente fónica; el niño es capaz de informar a su medio no solamente de lo que siente y quiere, sino de lo que piensa, empleando circuitos corticales de proyección y asociación frontoorbitales en conexión, a su vez, con las áreas perisilvianas. Es decir, se activan los circuitos neurofuncionales subcorticocorticales, talamocorticales, corticotalamicos y corticocorticales (áreas de asociación y comunicación empática).

En síntesis, la neuropsicología infantil permite comprender que las funciones cognitivas se encuentran en desarrollo durante la niñez. Estas funciones se construyen en base a los procesos biológicos que dirigen la maduración cerebral e interactúan con los factores medio ambientales. Así como los circuitos de nivel inferior maduran más pronto que los circuitos de nivel posterior, las funciones cognitivas que se forman primero sirven de base para el desarrollo de las siguientes.

Danese y Mcewen (2012) afirman que las experiencias adversas en la infancia se asocian con cambios permanentes en el sistema nervioso, el sistema endocrino y el sistema inmunitario. Estos cambios se pueden observar durante la infancia y siguen siendo evidentes en la vida adulta. Se han realizado innumerables estudios acerca de las efectos físicos y psicológicos del maltrato, sin embargo son pocos los estudios relacionados a los efectos neuropsicológicos y dentro de ellos el análisis de la percepción es aún insuficiente. En relación a la percepción visual Kale y Urbano (2013) encontraron que las señales faciales contienen información importante para guiar las interacciones sociales. Aunque no todos los humanos son igualmente expertos en el procesamiento de rostros, los factores genéticos y ambientales contribuyen a las diferencias en la capacidad de procesamiento de la cara.

En el caso de la violencia, Shenk, Putnam y Noll (2013), consideraron la relación entre el funcionamiento intelectual y la identificación facial. Advirtieron que las mujeres víctimas de maltrato con alto nivel intelectual lograban igual grado de reconocimiento facial que aquellas que no lo sufrían. Al analizar el caso de mujeres víctimas de maltrato con bajo nivel intelectual, se constató un menor rendimiento en el reconocimiento facial, en relación con las que no lo padecían. Estos resultados sugieren que en mujeres el maltrato y la inteligencia interactúan para predecir el reconocimiento de las emociones del rostro.

Los niños pequeños que se han visto expuestos a circunstancias traumáticas presentan también dificultades para identificar y responder a diferentes 
expresiones de las emociones y, por lo tanto, tienen problemas para establecer relaciones saludables (Wismer, et al., 2005). Por ejemplo, los niños que se han criado en hogares donde se ha producido maltrato físico muestran una sensibilidad exacerbada a las expresiones faciales de ira, lo que afecta negativamente a su comportamiento en entornos sociales desconocidos (Pollak, 2008). Por lo tanto, Fernández (2014) afirma que en la medida en que los niños contemplan el mundo como un lugar hostil y amenazante, es un potente factor de riesgo para la generación de problemas de comportamiento.

Los estudios acerca de la percepción auditiva se relacionan con tareas de análisis fonológico, las mismas que presentan puntuaciones significativamente inferiores (Fernández, 2014; Flores, Reyes, Aguilar, García y Cruzaley, 2012). Es innegable los efectos negativos del maltrato en el desarrollo infantil, sin embargo la literatura es pobre en analizar consecuencias específicas en la percepción, proceso que permite la interpretación de nuestro mundo. Por ello, este trabajo busca estudiar la percepción visual, auditiva y táctil en los niños víctimas de violencia intrafamiliar recién institucionalizados.

\section{Método}

\section{Participantes}

La muestra del estudio está constituida por 104 participantes. Uno de los grupos está conformado por niños víctimas de violencia intrafamiliar (NVVI) formado por escolares entre 5 y 12 años que llevan máximo tres meses institucionalizados a causa de la violencia intrafamiliar, y fueron ingresados en una casa de acogida de la ciudad de Cuenca (Ecuador) durante el periodo julio-diciembre de 2012. Se evaluó a un total de 52 niños y niñas, $48 \%$ mujeres y $52 \%$ varones, con una edad promedio de $8,2+1,51$ años. De los participantes de este grupo uno ha sufrido maltrato psicológico, uno maltrato psicológico y negligencia, seis han sido víctimas de maltrato psicológico, físico, sexual y negligencia, 21 psicológico, físico y negligencia y 23 maltrato psicológico y físico, según criterios del DSM 5.

Todos los participantes del grupo debían cumplir los siguientes criterios: tener entre 5 y 12 años; haber sido acogido en una de las casas por sufrir maltrato físico, y/o psicológico, y/o sexual, y/o negligencia de tipo intrafamiliar, al grado de que no pueden permanecer en su hogar de origen; los ni- 
ños debían estar acogidos en los últimos tres meses para evitar que el lenguaje no se encuentre mediatizado por el periodo de institucionalización y la evaluación no esté contaminada por procesos terapéuticos. Se excluyeron quienes presentan antecedentes de traumatismos cráneo-encefálicos severos, epilepsia, síndromes neurológicos, trastornos desintegrativos infantiles, psicosis, retraso mental u otra patología que le impida realizar la evaluación.

El segundo grupo de participantes de este estudio está formado por niños que viven con sus padres y que no presentan evidencia de violencia intrafamiliar, NNVI ( $56 \%$ mujeres y 44\% varones) con una edad promedio de $8,6+2,1$. Todos los participantes de este grupo debían cumplir los siguientes criterios: tener entre 5 y 12 años; estudiar en las mismas escuelas que los niños del grupo de estudio; equiparados en las características sociodemográficas. Al igual que en el grupo de NVVI se excluyen los niños que presentan antecedentes de traumatismos cráneo-encefálicos severos, epilepsia, síndromes neurológicos, trastornos desintegrativos infantiles, psicosis, retraso mental u otra patología que le impida realizar la evaluación.

\section{Instrumentos de medida}

Se aplicó las escalas y subescalas de percepción de la evaluación neuropsicológica infantil -ENI- de Matute, Rosselli, Ardila y Ostrosky (2007), en ellas se incluyen percepción táctil (mano derecha y mano izquierda); percepción visual (imágenes superpuestas de frutas, imágenes superpuestas de utensilios, imágenes superpuestas de juguetes, imágenes borrosas, cierre visual, reconocimiento de expresiones, integración de objetos) y percepción auditiva (notas musicales, sonidos ambientales, percepción fonémica). Se seleccionó esta prueba por estar validada para población latinoamericana, con altos coeficientes de correlación que estuvieron entre 0.858 y 0.987 .

\section{Procedimiento}

Para llevar a cabo el estudio se comunicó sobre esta investigación al Ministerio de Inclusión Económica y Social- Instituto de la Niñez y la Familia (MIES-INFA) y se solicitó una lista de las casas de acogida que se encuentran en la ciudad de Cuenca (Ecuador). Se obtuvo la autorización y colaboración de las autoridades de todas las casas de acogida en donde los niños 
cumplían con los criterios de inclusión. Una vez obtenido este permiso se informó a los responsables de los niños y se solicitó su consentimiento por escrito. A los niños se les solicitó su consentimiento oral y se les informó que podían abandonar la evaluación si así lo deseaban; se realizó a los niños una entrevista y junto con las historias clínicas se comprobó que cumplan los criterios de inclusión. Una vez aceptada su participación se aplicaron las pruebas de estudio. Por otra parte, para seleccionar el grupo de niños que no sufren de violencia intrafamiliar, se contactó con las escuelas a donde asisten los escolares del primer grupo. Se obtuvo la autorización verbal y escrita de las autoridades de las escuelas. Luego se envió una solicitud a los padres de familia, quienes dieron su autorización escrita y para corroborar los datos se revisaron las fichas de cada niño; también, se entrevistó a las maestras y/o personal del Departamento de Psicología, se hizo una primera entrevista y aquellos que cumplían los criterios de inclusión fueron invitados a participar en el estudio empírico y se aplicaron las pruebas.

\section{Análisis estadísticos}

Todos los análisis se realizaron con Statistical Package for Social Sciences (SPSS Inc., Chicago, IL), versión 22.0. Las variables no presentaron una distribución normal y homogeneidad $(\mathrm{p}<0,05)$ probadas a través de las pruebas de Kolmogorov-Smirnov (K-S) y el test Levene. Por ello y con la finalidad de evaluar la relación en los resultados de las tareas de lenguaje entre los NVVI y NNVI se realizó la U- de Mann-Whitney para las variables cuantitativas (Tabla 1)

\section{Resultados}

Los NVVI en la percepción táctil no muestran diferencias estadísticamente significativas, tanto en su componente de mano derecha como de mano izquierda $(\mathrm{p}>0,05)$.

En la percepción visual los NVVI presentan menor rendimiento sin diferencias estadísticamente significativas en las sub escalas de imágenes superpuestas, imágenes borrosas, cierre visual e integración de objetos $(\mathrm{p}>0,05)$; sin embargo presentan menor rendimiento con diferencias estadísticamente significativas en la tarea de reconocimiento de expresiones $(U=921,5 ; p=<0,01)$. 
Con relación a la percepción auditivas no presentan diferencias estadísticamente significativas en la subescala de percepción de notas musicales ( $\mathrm{p}>0,05)$; pero si en las subescalas de percepción de sonidos ambientales $(U=1033 ; p<0,05)$ y en percepción fonémica $(U=1018 ; p=<0,05)$. Como se observa en la tabla 1.

\section{Tabla 1}

\section{Habilidades perceptuales. Descriptivos y comparación entre grupos}

\begin{tabular}{|c|c|c|c|c|c|c|c|c|}
\hline \multirow[b]{2}{*}{$\begin{array}{l}\text { HABILIDADES } \\
\text { PERCEPTUALES }\end{array}$} & \multicolumn{2}{|c|}{ NVVI $(n=52)$} & \multirow[b]{2}{*}{ RIQ } & \multicolumn{2}{|c|}{ NNVI $(n=52)$} & \multirow[b]{2}{*}{ RIQ } & \multirow[b]{2}{*}{$\mathbf{U}$} & \multirow[b]{2}{*}{ p } \\
\hline & $\begin{array}{l}\text { Mín- } \\
\text { Máx } \\
\end{array}$ & Mdn & & $\begin{array}{l}\text { Mín- } \\
\text { Máx }\end{array}$ & Mdn & & & \\
\hline \multicolumn{9}{|l|}{ Percepción táctil } \\
\hline Mano derecha & $6-8$ & 8 & $7-8$ & $7-8$ & 8 & $8-8$ & 1243 & 0,332 \\
\hline Mano izquierda & $7-8$ & 8 & $7,25-8$ & $7-8$ & 8 & $8-8$ & 1164 & 0,068 \\
\hline \multicolumn{9}{|l|}{ Percepción visual } \\
\hline Imágenes superpuestas de frutas & $1-6$ & 4 & $3-5$ & $0-6$ & 4 & $4-5$ & 1257 & 0,524 \\
\hline Imágenes superpuestas de utensilios & $0-5$ & 3 & $3-4$ & $0-6$ & 4 & $3-4$ & 1092 & 0,078 \\
\hline Imágenes superpuestas de juguetes & $0-4$ & 3 & $2-3$ & $0-5$ & 3 & $2-3$ & 1214,5 & 0,346 \\
\hline Total imágenes superpuestas & $4-14$ & 10 & $8-11$ & $0-14$ & 10,5 & $9-12$ & 1078,5 & 0,073 \\
\hline Imágenes borrosas & $3-10$ & 7 & $5-8$ & $2-10$ & 6 & $5-8$ & 1218 & 0,378 \\
\hline Cierre visual & $0-7$ & 4 & $2-5$ & $1-7$ & 4 & $3-5$ & 1235 & 0,439 \\
\hline Reconocimiento de expresiones & $2-8$ & 6 & $5-7$ & $4-8$ & 7 & $6-8$ & 921,5 & $0,004^{* *}$ \\
\hline Integración de objetos & $0-6$ & 3 & $2-4$ & $0-7$ & 3 & $2-4$ & 1233 & 0,429 \\
\hline \multicolumn{9}{|l|}{ Percepción auditiva } \\
\hline Notas musicales & $0-8$ & 4 & $4-5$ & $2-8$ & 5 & $4-6$ & 1184,5 & 0,263 \\
\hline Sonidos ambientales & $0-7$ & 4 & $4-5$ & $2-8$ & 5 & $4-5$ & 1033 & $0,030^{*}$ \\
\hline Percepción fonémica & $1-20$ & 18,50 & $15-19$ & $1-20$ & 19 & $17-20$ & 1018 & $0,026^{*}$ \\
\hline
\end{tabular}

NVVI: Niños Víctimas de Violencia Intrafamiliar; NNVI: Niños que no han sufrido Violencia intrafamiliar.

Mín: Mínimo; Max: Máximo; Me: Mediana; RIQ: Rango intercuartil; U (U-MannWhitney). 


\section{Discusión}

$\mathrm{Al}$ analizar las habilidades perceptivas, no se obtuvieron mayores diferencias en la percepción táctil (mano derecha e izquierda) y la percepción visual (imágenes superpuestas de frutas, utensilios, juguetes; imágenes borrosas, cierre visual e integración de objetos). La percepción de objetos según Fox et al. (2010) se inicia en la formación del sistema visual en la etapa pre-natal, oscilando hasta más allá de los diez primeros años de vida. Inclusive, existe un período ulterior de estabilización para poder mantener el nivel de competencia adquirido. Este largo período de desarrollo permite que el sistema visual del niño se adapte a las características específicas de su ambiente. Lo que facilita a los NVVI la adaptación de los procesos de percepción visual.

Sin embargo, es evidente el menor rendimiento del grupo de NVVI en la tarea de reconocimiento de expresiones. La diferencia entre la percepción de objetos y de caras fue descrita por O'Riordan, Plaisted, Driver (2001), quienes evaluaron a individuos que exhiben un procesamiento alterado en el reconocimiento de caras y preservado de objetos; además, la evidencia de diferentes estructuras cerebrales que se activan durante el procesamiento de caras y objetos, pero con una cercanía anatomofuncional (Prvulovic, Hubl y Sack, 2002). El rendimiento por debajo de lo esperado en la tarea de reconocimiento de expresiones corrobora que la exposición al maltrato en la infancia se asocia con alteraciones en la capacidad de procesamiento de rostros y su función social, todo ello relacionado con una mayor sensibilidad a una amenaza potencial (Kale y Urbano, 2013).

En relación a la audición, los neonatos son capaces de distinguir todos los sonidos de todos los idiomas del mundo, aunque no los hayan oído nunca antes. Sin embargo, al terminar el primer año de vida, solamente conservan la habilidad de distinguir los sonidos que han escuchado en las conversaciones de las personas que los rodean (Werker y Tees, 2005). Esto explica que en nuestro estudio los NVVI presentan un déficit en la percepción de sonidos ambientales y fonémicos. Además, se relaciona con dificultades en el análisis fonológico (Fernández, 2014; Flores, Reyes, Aguilar, García y Cruzaley, 2012). Sin embargo, en la percepción de notas musicales no existe esta notoria diferencia por la falta de un ambiente estimulante para la educación musical de ambos grupos de estudio.

En estos niños se encuentra conservada la percepción táctil; la percepción visual es adecuada excepto cuando se involucra el componente emo- 
cional; y en lo auditivo existe déficit en percepción de sonidos ambientales y verbales.

\section{Conclusiones y recomendaciones}

Los Niños Víctimas de Violencia Intrafamiliar se caracterizan por sufrir un tipo de violencia transgeneracional que les afecta desde las primeras etapas del desarrollo del infante y por ende existe un impacto directo sobre sus funciones cognitivas. Dentro de ellas la percepción es aquella que nos permite tener un contacto directo con nuestro medio y una de las primeras en desarrollarse, en este estudio se observa que la violencia intrafamiliar afecta de diversas maneras a estas funciones.

La percepción táctil no se encuentra alterada, en relación a la percepción visual se observa un buen funcionamiento, excepto cuando se involucran las emociones, lo que nos indica las dificultades de los NVVI en aprovechar oportunidades que se presentan en la vida pues están orientados siempre a sobre identificar aspectos negativos. En lo concerniente a la audición se observa un déficit en los procesos básicos para la lecto escritura como son la percepción en sonidos ambientales y fonémicos.

Al estar alterados procesos básicos en el desarrollo cognitivo de los niños Víctimas de Violencia Intrafamiliar se recomienda que los centros de acogida integren en sus protocolos de actuación la evaluación y rehabilitación neuropsicológica.

Además, a pesar de los esfuerzos realizados para erradicar la violencia se observa un aumento de la misma y las estadísticas son realmente preocupantes. Por ello a más de buscar mayor agilidad en la aplicación de sanciones a los agresores, las políticas públicas debería incorporar nuevas formas de apoyo al bienestar en el ámbito familiar, núcleo de la sociedad, que permita un desarrollo armónico infantil.

\section{Bibliografía}

Astle, D.E. \& Scerif, G. (2011). Interactions between attention and VSTM: Lessons from developmental and individual differences. Neuropsychologia, 49, $1435-45$. 
Bezrukikh, M., Machinskaya, R., \& Farber, D. (2009). Structural and functional organization of a developing brain and formation of cognitive functions in child ontogeny. Human Physiology, 35(6), 658-671. doi:10.1134/ S0362119709060024.

Calle, H. (2012). Violencia y maltrato en niños, niñas y adolescentes. Cuenca: Concejo Cantonal de la Niñez y Adolescencia de Cuenca y Facultad de Jurisprudencia y Ciencias Políticas y Sociales de la Universidad de Cuenca.

Congreso Nacional del Ecuador (2008). El plenario de las Comisiones Legislativas. Ley contra la violencia a la mujer y la familia. Quito, Ecuador.

Consejo Cantonal de la Niñez y Adolescencia de Cuenca y Alcaldía de Cuenca (2013). Guía de Entidades y Servicios para la Niñez y Adolescencia del cantón Cuenca.

Corsi, J. (2006). Maltrato y abuso en el ámbito doméstico. Buenos Aires: Paidós.

Danese, A., \& Mcewen, B. S. (2012). Adverse childhood experiences, allostasis, allostatic load, and age-related disease. Physiology \& Behavior, 106(1), 29-39. doi:10.1016/j.physbeh.2011.08.01.

Fernández, V. (2014). Maltrato infantil: Un estudio empírico sobre variables psicopatológicas en menores tutelados. Tesis doctoral, Universidad de Murcia.

Flores, M., Reyes, V., Aguilar A., García C., \& Cruzaley A. (2012). Desarrollo de los factores neuropsicológicos asociados a la lectoescritura en niños víctimas de violencia física. Integración Académica en Psicología, 1(3), 82-88.

Fox, S., Levitt, P., \& Nelson, C.A. (2010). How the timing and quality of early experiences influence the development of brain architecture. Child Development, 81, 28-40.

Fox, N., \& Shonkoff, J. (2011). Modo en que el temor y la ansiedad persistentes pueden afectar al aprendizaje, al comportamiento y a la salud de los niños pequeños. En: Violencia oculta protegiendo a los niños pequeños en el hogar. Espacio para la Infancia, 36, 8-14. Fundación Bernard Van Leer.

Gavilanes, R. (2008). Sistemas de atención infantil en el Ecuador. Quito: Abya-Yala.

Giza, C., Mink, R., \& Madikians, A. (2007). Pediatric traumatic brain injury: not just little adults. Current opinion in critical care, 13(2), 143-52.

Gunnar, M. R., Talge, N. M., \& Herrera, A. (2009). Stressor paradigms in developmental studies: What does and does not work to produce mean increases in salivary cortisol. Psychoneuroendocrinology, 34(7), 953-967. doi:10.1016/j.psyneuen.2009.02.010. 
Heckman, J. (2007). The economics, technology, and neuroscience of human capability formation. Proceedings of the National Academy of Sciences, 104(3), 13250-5.

Johnson, M. H. (2011). Developmental Cognitive Neuroscience Interactive Specialization: A domain-general framework for human functional brain development? Accident Analysis and Prevention, 1(1), 7-21. doi:10.1016/j. den.2010.07.003.

Kale, E., \& Urbano, J. (2013). Psychiatry Research: Neuroimaging Childhood maltreatment and response to novel face stimuli presented during functional magnetic resonance imaging in adults. Psychiatry Research: Neuroimaging, 212(1), 36-42. doi:10.1016/j. pscychresns.2012.11.009.

Knudsen, E.I., Heckman, J.J., Cameron, J.L., \& Shonkoff, J.P. (2006). Economic, neurobiological, and behavioral perspectives on building America's future workforce. Proceedings of the National Academy of Sciences, 103, 10155-62.

Lebel, C., \& Beaulieu, C. (2011). Longitudinal development of human brain wiring continues from childhood into adulthood. Journal of Neuroscience, 31, 10937-47.

Litrownik, A., Newton, R., Mitchell, B., \& Richardson, K. (2003). Long-Term Follow-Up of Young Children Placed in Foster Care: Subsequent Placements and Exposure to Family Violence. Journal of Family Violence, 18(1). doi: 10.1023/A:1021449330344.

Luria, A. R. (1978). Cerebro y lenguaje. Barcelona: Fontanella.

Manga, D., \& Fournier, C. (1997). Neuropsicología clínica infantil. Madrid: Editorial Universitas.

Manga, D., \& Ramos F. (2011). El legado de Luria y la neuropsicología escolar, Psychology, Society, \& Education, 3 (1),1-13

Mattick, J.S., \& Mehler, M.F. (2008). RNA editing, DNA recording and the evolution of human cognition. Trends in Neurosciences, 31(5), 227.

Matute, E., Rosselli, M., Ardila, A., \& Ostrosky-Solís, F. (2007). Evaluación neuropsicológica infantil. México: Manual Moderno.

Morelato, G. (2011). Maltrato infantil y desarrollo: hacia una revisión de los factores de resiliencia. Pensamiento psicológico, 9(17), 83-96.

Muñoz, J., \& Palau M. (2004) Ontogenia de la autoconciencia como se construye un cerebro cognitivo. Revista de Neurología, 38 (1), S3-S8.

National Scientific Council on the Developing Child (2010). Early Experiences Can Alter Gene Expression and Affect Long-term Development. Working 
Paper n. ${ }^{\circ}$ 10. Cambridge, MA: National Scientific Council on the Developing Child.

Oates, J., Karmiloff-Smith, A., \& Johnson, M. (2012). El cerebro en desarrollo. La primera infancia en perspectiva. Reino Unido: The Open University.

Obando, O. L., Villalobos, M. E., \& Arango, S. L. (2010). Resiliencia en niños con experiencias de abandono. Acta Colombiana de Psicología, 13(2),149-159.

Ojeda, L. (2010). Violencia, delincuencia e inseguridad en el Ecuador. Quito: Universidad Alfredo Pérez Guerrero.

O’Riordan, M., Plaisted, K., \& Driver, J. (2001). Sulperior visual search in autism. Journal of Experimental Psychology and a Human Perception and Performance, 27, 719-730

Organización Mundial de la Salud (2014). Maltrato infantil. Nota descriptiva No. 150. Recuperado de http://who.int/mediacentre/factsheets/fs150/es/

Organización Panamericana de la Salud para la Organización Mundial de la Salud (2002). Informe mundial sobre la violencia y la salud. Washington: OPS. http://www.unicef.org/violencestudy/reports/SG_violencestudy_sp.pdf

Prvulovic, D., Hubl, D., \& Sack, A. (2002) Functional imaging of visuoespatial processing in Alzheimer's disease. Neuroimage, 17, 1403-1414.

Pollak, S.D. (2008). Mechanisms linking early experience and the emergence of emotions: illustrations from the study of maltreated children. Current $D i$ rections in Psychological Science, 17, 370-375

Quintanar L., Solovieva, Y., Azcoaga, J., Peña, E., Bonilla, M., \&Yáñez, G. (2008). Los trastornos del aprendizaje: Perspectivas neuropsicológicas. Bogotá: Cooperativa Editorial Magisterio.

Rosselli, M., Matute, E., \& Ardila, A. (2010). Neuropsicología del desarrollo infantil. México: Manual Moderno.

Shenk, C. E., Putnam, F. W., \& Noll, J. G. (2013). Predicting the accuracy of facial affect recognition: the interaction of child maltreatment and intellectual functioning. Journal of Experimental Child Psychology, 114(2), 229-42. doi:10.1016/j.jecp.2012.08.007

Shonkoff, J.P. \& Richter, L. (2013). The powerful reach of reach of child development: a science-based foundation for sound investment. En: P.R. Britto, P. Engle, y C. Super (Eds.), Handbook of Early Childhood Development Research and its Impact on Global Policy. Nueva York, NY: Oxford University Press.

SENPLADES. (2012). Acogimiento Familiar. Sistema Nacional de Información del Ecuador. Recuperado de http://app.sni.gob.ec/web/menu/ Acogimiento Familiar. 
Sur, M. \& Rubenstein J.L. (2005). Patterning and plasticity of the cerebral cortex. Science, 310, 805-10.

Tsavoussis, A., Stawicki, S. P., \& Papadimos, T. J. (2015). Child-witnessed domestic violence: An epidemic in the shadows. International Journal of Critical Illness and Injury Science, 5(1), 64-65. doi:10.4103/2229-5151.152354.

UNICEF (2014). Ocultos a plena luz del día. Un análisis estadístico de la violencia contra los niños (resumen).

http://www.unicef.org/lac/VR_Exec_Summary_8_29_SP(3).pdf

Vygotsky, L. (2001/1982d). El problema del lenguaje y el pensamiento del niño en la teoría de Piaget. En L. Vigotski, Obras Escogidas (A. Alvarez, \& P. Del Río, Trads., Vol. II, págs. 29-80). Madrid: A. Machado Libros.

Vygotsky, L. S. (1982/1997i). Obras escogidas: La psique, la consciencia y el inconsciente. En: L. S. Vygotsky, y A. Zaporozhéts (Eds.), Obras escogidas: Problemas teóricos y metodológicos de la Psicología. (A. Alvarez, \& P. Del Río, Trads., 2a ed., Vol. I, págs. 95-110). Madrid, España: Visor.

Wismer, A.B., Ziegler, T.E., Kurian, J.R., Jacoris, S. \& Pollak, S.D. (2005). Early experience in humans is associated with changes in neuro-peptides critical for regulating social behavior. Proceedings of the National Academy of Sciences USA, 102(47), 17.237-17.240.

Werker, J.F. \& Tees, R.C. (2005). Speech perception as a window for understanding plasticity and commitment in language systems of the brain. Developmental Psychobiology, 46, 233-61. 